\title{
Papillon-Lefevre Syndrome with Aggressive Periodontitis - A Case Report with Review of Literature
}

\author{
Authors \\ Dr Suryakanth Malgikar*1, Dr Madanika $\mathbf{P}^{\mathbf{2}}$, Dr Venkatesh Suneel $\mathbf{B}^{\mathbf{3}}$ \\ ${ }^{1}$ Senior Lecturer, Department of Periodontology, KIMS Dental College, Amalapuram \\ ${ }^{2}$ Assistant Professor, Department of Biochemistry, KIMS \& RF, Amalapuram \\ ${ }^{3}$ Professor \& HOD, Department of Prosthodontics, KIMS Dental College, Amalapuram \\ Corresponding Author
}

Dr Suryakanth Malgikar*

Senior Lecturer, Department of Periodontology, KIMS Dental College, Amalapuram - 533201, East

Godavari District, Andhra Pradesh

Email: drmalgikarsuryakanth@gmail.com, Mobile+91-9591212165

\begin{abstract}
Papillon-Lefevre Syndrome (PLS) is a rare autosomal recessive disorder first described by two French physicians, Papillon and Lefevre in 1924. Papillon-Lefevre Syndrome (PLS) is described as the association of palmar-plantar hyperkeratosis with precocious periodontal disease which results in exfoliation of primary and permanent dentitions. The majority of the teeth are lost by the age of 14 to 15 years. This case report describes the periodontal findings and dermatological findings with review of literature.

Keywords: Papillon-Lefevre syndrome, Palmoplantar hyperkeratosis, Keratodermas, Rapidly progressing periodontitis.
\end{abstract}

\section{INTRODUCTION}

Rapid and advanced destruction of periodontal tissues in early childhood has been reported both in systemically healthy children and in children with systemic diseases such as hypoposphatasia, neutropenia, diabetes mellitus, leukocyte adhesion deficiency syndrome, Down's syndrome, EhlersDanlos syndrome Type VIII, and Papillon-Lefevre syndrome. ${ }^{1}$ In 1924, Papillon and Lefèvre ${ }^{1}$ described 2 siblings in a family with consanguinity exhibiting the association of palmar-plantar hyperkeratosis with precocious periodontal disease that resulted in exfoliation of primary and permanent dentitions. In this entity, examination of the periodontal tissues reveals severely inflamed gingiva, deep periodontal pockets, diffuse and extensive bone resorption, and loosening and exfoliation of the teeth. An increased susceptibility to infections has also been reported in patients with PLS. ${ }^{2}$ Affected patients show palmoplantar keratoderma manifestations consisting of hyperkeratosis of palms, soles of the feet, elbows, knees, and other organs, as well as a rapidly progressive, severe periodontitis that leads to early loss of deciduous and permanent teeth. Genetic studies ${ }^{3}$ have revealed mutations in the cathepsin C gene (CTSC) that are believed to be the principal etiologic factor of this disease.

This case report describes the clinical presentations in a case with typical dental and 
dermatological findings. Periodontal management and therapeutic treatment of a patient diagnosed as Papillon-Lefevre syndrome with aggressive periodontitis.

\section{CASE REPORT}

A 15 year old male reported to the Department of Periodontics, with the chief complaint of having lost most of his teeth and inability to chew with the remaining 'loose' teeth. Medical history revealed that the patient had been suffering from recurrent skin infections since an early age with thickening and subsequent peeling of the skin of his hands and feet, for which he had been undergoing intermittent treatment. His grandfather had similar dermatological lesions. His parents noted that he had recurrent abscesses healing with scars since early infancy. Past dental history revealed that the patient had lost all his deciduous teeth by about 3 years of age. A number of his permanent teeth also became mobile soon after eruption and were subsequently extracted (Figure: 1). The panoramic radiograph showed loss of several teeth with severe alveolar bone loss (Figure: 2).

He had lost all his deciduous teeth due to chronic swelling and bleeding of the gums until the age of 2 years. He had no family history of similar dermatological findings but history revealed third degree consanguinity in his parents. On dermatological examination there were multiple fissured erythematous hyperkeratotic plaques on the soles (Figure: $3 \& 4$ ), Xerosis and erythema on the palms and dispersed erythematous papules on the trunk (Figure: 5).

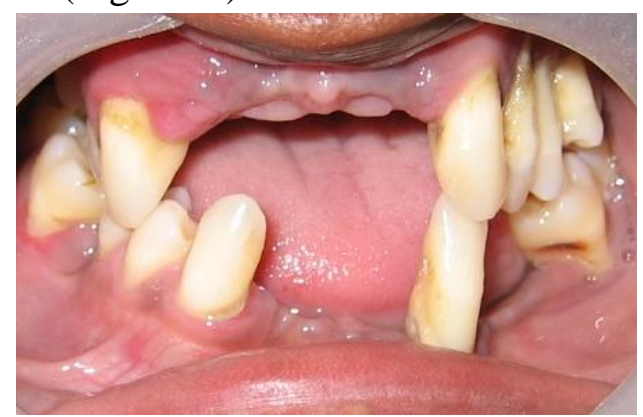

Figure: 1 Clinical appearance with severe gingival inflammation and loss of several teeth

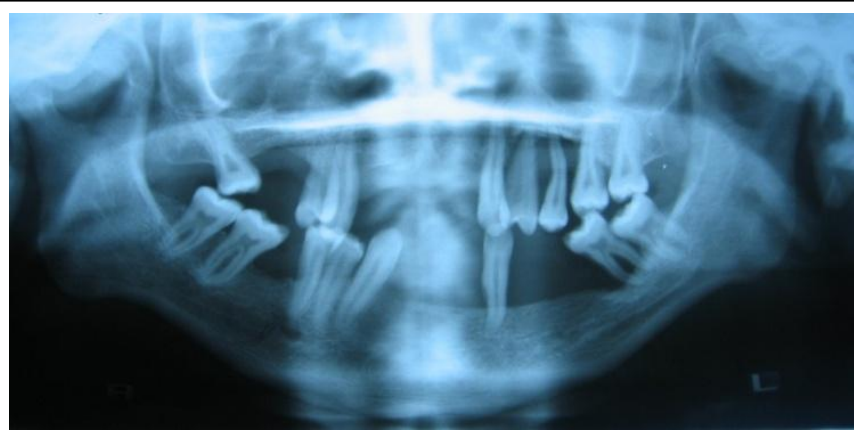

Figure: 2 Panoramic radiograph showing loss of several teeth and severe alveolar bone loss

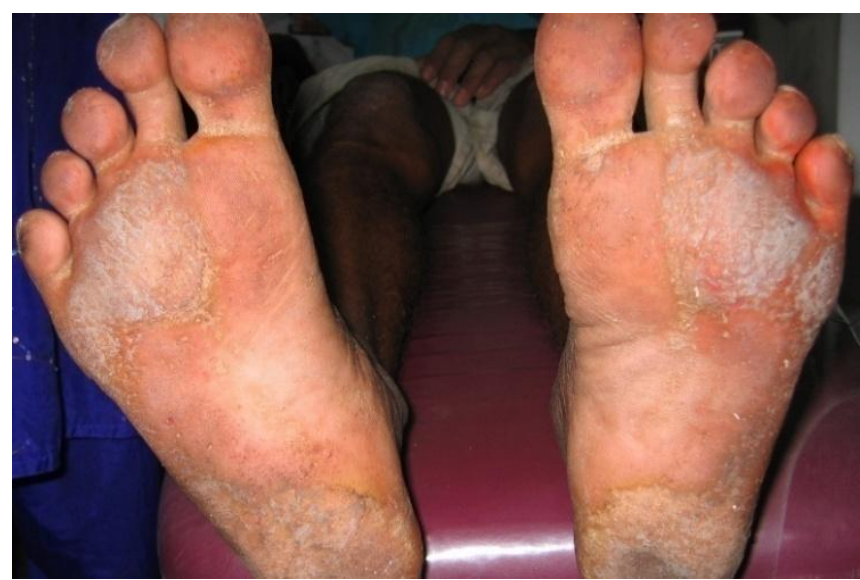

Figure: 3 Keratotic plaques on the ventral surface of right and left foot

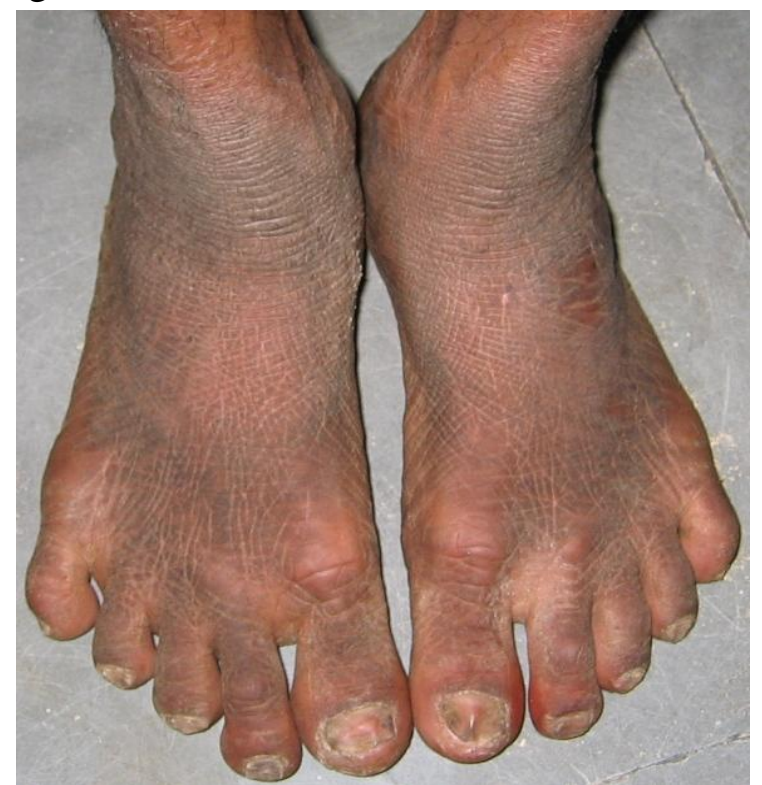

Figure: 4 Keratotic Plaque on right and left foot 


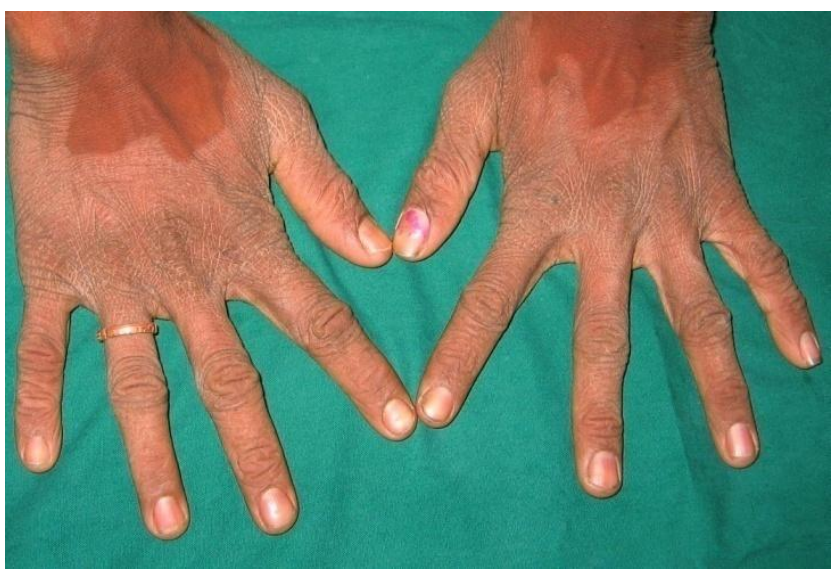

Figure: 5 Keratotic Plaque on the dorsal surface of right and left hand

\section{DISCUSSION}

Haneke $^{4}$ used the following three criteria to classify a case as PLS: (a) palmoplantar hyperkeratosis; (b) loss of primary and permanent teeth; and (c) autosomal recessive inheritance. The exact etiology of Papillon-Lefevre syndrome is still obscure; however, genetic, immunologic, and microbiologic factors have all been linked to the development of the syndrome. Neutrophil is the key protective cell in the defense of periodontal tissues. The decreased chemotaxis of peripheral blood neutrophils strongly suggests that the neutrophils may act as one of the important determinants of the pathogenesis of the severe periodontal destruction in patients with PLS. The immunological status of the disease has been investigated in a limited number of case reports. It was found that, although there is no statistically significant variation in the distribution of $\mathrm{CD}_{4}+$ and $\mathrm{CD}_{8}+$ lymphocytes and $\mathrm{Th} / \mathrm{Ts}$ ratio, $\mathrm{CD}_{29}{ }^{+}$ cells were increased while CD45RA+ cells were decreased in patients with Papillon-Lefevre syndrome. $^{2}$

Recently, genetic examinations point to the Papillon- Lefevre syndrome gene locus in these patients. The gene responsible for it has been localized to chromosome 11q14-q21 where the cathepsin $\mathrm{C}$ gene codes for a lysosomal protease, in the interval between D11S4082 and D11S931. Subsequently, inactivating mutations were identified in this gene and an almost total loss of cathepsin $\mathrm{C}$ activity was shown in patients with
Papillon-Lefevre syndrome. Mechanistically, deficient activation of leukocyte serine proteinases due to lack of cathepsin $\mathrm{C}$ gene activity could possibly explain the severe periodontitis in PLS. ${ }^{\mathbf{5}}$ Several bacterial species have been identified in the subgingival plaque of patients with PLS, including Aggregatibacter actinomycetemcomitans, Porphyromonas gingivalis, Tannerella forsythia, Parvimonas micra and other species. ${ }^{\mathbf{6}}$

\section{CONCLUSION}

It is important that dentists be familiar with the aetiology of Papillon-Lefevre syndrome, as well as with Papillon-Lefevre Syndrome specific dermatologic manifestations and periodontal involvement, to be able to identify the disease in its very early stages. Even though PLS is an extremely rare condition it is associated with lifelong psychological and social impacts on the growing children such as depression including hopelessness, aimlessness, social phobia, and a fear of communicating with people outside their family. Combined cooperation from dermatologists, pediatricians, periodontists, and prosthodontists is critical for the overall care of patients suffering from PLS.

\section{REFERENCES}

1. Papillon MM, LeFevre P. Deux cas de keratodemie palmaire et plantaire symetrique familiale (Malide de Meleda) chez le frere et la soeur. Coexistence dens le deux d'alterations dentaires graves. Bull Soc Fran Dermatol Syphil 1924;31: 82-84.

2. Erhan Firatli, Binnur Tüzün, and Ahmet Efeo lu. Papillon-Lefevre Syndrome. Analysis of Neutrophil Chemotaxis. J Periodontol 1996; 67: 617-620.

3. Hart PS, Zhang Y, Firatli E, et al. Identification of cathepsin $\mathrm{C}$ mutations in ethnically diverse papillon-Lefevre syndrome patients. J Med Genet 2000; 37: 927-932.

4. Haneke E. The Papillon-Lefevre syndrome: keratosis palmoplantaris with 
periodontopathy. Human Genetics 1979; 51: 1-35.

5. Kaustubh Suresh Thakare, M. L. Bhongade, Pretti Charde, Shweta Kale, Priyanka Jaiswal, B. K. Somnath, and Sunil Pendor. Genetic Mapping in Papillon-Lefevre Syndrome: A Report of Two Cases. Case Reports in Dentistry. Volume 2013.

6. Jasim M. Albandar, Razan Khattab, Fawza Monem, Sara M. Barbuto, and Bruce J. Paster. The Subgingival Microbiota of Papillon-Lefevre Syndrome. J Periodontol 2012; 83: 902-908. 